\title{
The use of expanded polytetrafluoroethylene in depressed deformities of the face
}

\author{
XIAOWEI LIU, YIMING ZHANG, SHAOLIANG WANG, ZEYUAN LEI, XIANG LI and DONGLI FAN \\ Department of Plastic Surgery, Xinqiao Hospital, Third Military Medical University, Chongqing 400037, P.R. China
}

Received December 11, 2015; Accepted June 3, 2016

DOI: 10.3892/etm.2016.3771

\begin{abstract}
Expanded polytetrafluoroethylene (ePTFE) has been extensively used for facial soft tissue augmentation procedures, and is regarded as safe and reliable and suitable as a permanent implant. This implant is generally used in the lower third of the face for lips filling, nasal augmentation, nasolabial folds and chin augmentation, and rarely for congenital or acquired depressed deformities of the face. The aim of the present study was to assess the effects of ePTFE in congenital or acquired depressed deformities of the face. From September, 2008 to January, 2014, 26 patients were implanted with the material ePTFE to correct depressed deformities of the face. The average age at operation was 23.2 years, with a range of 17-45 years. The depressed deformities were lateralized. The follow-up period was 6-18 months (average 9 months). The etiologies of the depressed deformities included stable hemifacial atrophy (3 cases), craniofacial microsomia (13 cases), bony depression after trauma (8 cases), and other unclear reasons ( 2 cases). The operations were performed under local anesthesia. ePTFE was inserted in different tissue planes that varied among the different subanatomical areas in the face: beneath the superficial temporal fascia in the temporal area, and on the surface of the superficial musculoaponeurotic system in the zygomatic area, cheek and mandibular area. All of the patients were followed up. Most of the patients [25 of 26 patients (96.2\%)] were satisfied with the results, while 1 patient $(3.8 \%)$ was not satisfied for incomplete correction of the depressed deformity. In conclusion, aside from lipofilling and a free flap transfer, the results showed that ePTFE was an alternative treatment for facial depressed deformity.
\end{abstract}

\section{Introduction}

Contour enhancements of the face constitute an important aspect of facial plastic surgeries for cosmetic, as well as

Correspondence to: Dr Dongli Fan, Department of Plastic Surgery, Xinqiao Hospital, Third Military Medical University, 1 Xinqiaozheng Street, Chongqing 400037, P.R. China

E-mail: pgp172@163.com

Key words: facial depressed deformity, facial augmentation, expanded polytetrafluoroethylene, biomaterials traumatic, congenital, and extirpative defect corrections (1). There are various options to consider for the reconstruction of a facial depressed deformity depending on whether the underlying defect is a skeletal or a soft tissue depression, including autologous tissue grafts, allogenic tissue grafts and alloplastic materials.

Autologous tissues, such as grafted adipose tissue (lipofilling), are thought to produce a more natural reconstruction of the contour of the face, but they are highly invasive with significant donor site morbidity and have drawbacks including limited availability, limited moldability, and unpredictable resorption (2). These disadvantages constrict their clinical use. Various alloplastic materials have been used in facial cosmetic and reconstructive surgery. The material should have good biocompatibility, be easy to remodel at the operating table, maintain its desired form and consistency in situ, and be inert in body tissue (3). The most commonly used materials in clinical practice include silicone, Gore-Tex, medpore, and expanded polytetrafluoroethylene (ePTFE) $(1,2)$. Silicone implants lack the ability for vascularization, promote thick capsule formation, cause resorption of the underlying bone, and display a tendency for the implant to shift or extrude over a long period of time. With Gore-tex the risk of infection, seroma formation, and shifting from optimal place increases. Medpor has good biocompatiblility, but due to its characteristic of stiffness, it is mainly used on the bone reconstruction and cannot be used in the soft tissue.

W.L. Gore and Associates, Inc. first produced ePTFE in $1969(4,5)$. The material was first used in 1982 to reconstruct soft-tissue deficiencies. Since then, ePTFE has been used safely and effectively in the human body for various applications in vascular and cosmetic surgery. In cosmetic surgery, ePTFE was often used for facial wrinkles, and for chin and nasal augmentation.

In the present study, the ePTFE was used for large-area facial depressed deformities. The results showed that, ePTFE constituted a viable alternative treatment for facial depressed deformity.

\section{Patients and methods}

Patient selection. In theory, any healthy patient with a facial depressed deformity is suitable for ePTFE implantation. However, to obtain a more satisfactory outcome for the surgeon and the patient, patient selection should be deliberate. An 
ePTFE implant is more suitable for patients with thick skin and subdermal tissue, and especially for patients who do not want to undergo a more invasive surgery of free-flap transfer. ePTFE is a long-lasting solid implant that is palpable and visible on animation when it is implanted in thin subdermal tissue.

In the present study, 31 ePTFE implants were used for facial augmentation in 26 patients (12 women, 14 men) between September 2008 and January 2014. The patient age range was $17-45$ years (mean age 23.2 years). Indications for the augmentation procedure were congenital malformations, post-traumatic defects, and reconstruction after tumor surgery. Of the 26 patients, diagnoses included stable hemifacial atrophy ( 3 cases), craniofacial microsomia (13 cases), bony depression after trauma (8 cases), and other unclear reasons for soft tissue atrophy ( 2 cases). The implants were used for augmentation in the nasal/paranasal area, zygomatico-orbital area, and chin and mandibular area. The postoperative follow-up periods ranged from 6 to 18 months (average 9 months).

Surgical technique. The procedures were performed under local anesthesia (a solution of $0.5 \%$ lidocaine with 1:200,000 epinephrine). The depressed deformity area was marked with methylene blue, and an ePTFE implant with the proper thickness, was trimmed on the edges for a polished smooth transition effect.

For the frontal area, the surgeon first marked the region to be treated with an indelible marker. If the patient had displayed a strong frontalis movement during the pre-operatory examination, the botulinum toxin was injected 1 week before the implanting procedure. After marking, a solution of 0.5\% lidocaine and 1:200,000 epinephrine was infiltrated along the planned dissection area and the two supraorbital ridges to block the supratrochlear and supraorbital nerves. A no. 15 Bard-Parker blade (Aspen Surgical Products, Inc., Chicago, IL, USA) was used to make an incision along the $5-\mathrm{cm}$ in length marked line behind the anterior hairline down through the level of the periosteum. A subperiosteal dissection was carefully made, inferiorly to the level of the superior orbital rim and laterally to the temporal ridge, taking care not to tear the periosteum or cross over the temporal fusion line, and carefully avoiding the supraorbital neurovascular bundles. The range of dissection extended into normal tissue $0.5-1 \mathrm{~cm}$ beyond the junctional transition zone of the defect. The trimmed ePTFE implant was inserted into the subperiosteal pocket with utmost precision and the edges were inspected carefully to confirm that there was no buckling or folding. Then, the incision was sutured.

For the cheek, temporal, and mandibular areas, the procedure was almost the same except for the obvious differences regarding the tissue plane for implanting and the access sites. For patients with a recipient site on the cheek and zygomatic areas, a preauricular incision was made to create a pocket between the subcutaneous tissue and the superficial musculoaponeurotic system (SMAS). For the temporal and frontal depressed deformities, an incision behind the hairline was selected, the dissected space lay between the deep and superficial temporal fascias, and the utmost care was taken to preserve the facial nerve intact. For augmentation of the mandibular area, a submental incision was made to access the implantation site (Fig. 1).

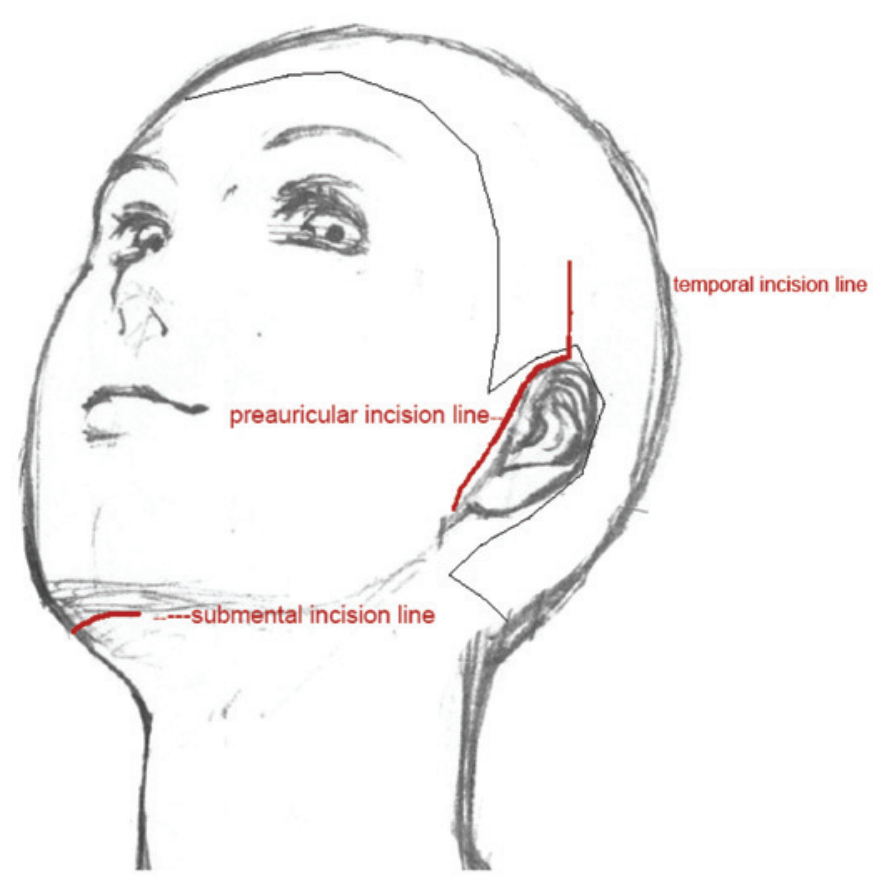

Figure 1. Diagram of incisions for expanded polytetrafluoroethylene implanting.

\section{Results}

In order to treat facial depression, 31 ePTFE were implanted into the temporal areas (3 implants), cheek areas (5 implants), zygomatic areas (10 implants), mandibular areas (8 implants) and frontal areas (5 implants) in 26 patients.

No major complications occurred during the follow-up of the 26 patients who had ePTFE implants inserted for varied depressed deformities in the frontal, temporal, zygomatic, and mandibular areas. There were no cases of infection, implant exposure, delayed hematoma, or seroma. Minor complications, such as immediate postoperative hematoma, visible or palpable lateral border, asymmetries of shape, visible scars, or hair loss were also rare. There was 1 patient (3.8\%) with an under-corrected depressed contour, while the remaining 25 patients were satisfied with the outcomes (satisfaction rate, 96.2\%) (Figs. 2 and 3).

\section{Discussion}

ePTFE is a woven form of PTFE that creates a mesh-like structure. It is flexible, soft and strong, non-toxic, biocompatible, and not water-soluble. It has been reported that although the ePTFE soft-tissue patch is a porous material (average pore size is $22 \mu \mathrm{m}$ ), it does not appear to allow extensive fibrous tissue ingrowth, as do other porous alloplastic materials $(4,5)$. Previous findings have shown that the pores of ePTFE provide a lattice for incorporating connective tissue (6). The phenomenon of little to no tissue adhesion allows for easy removal in case of complication or if the patient is not satisfied with the augmentation result. The level of tissue reaction to ePTFE is little, and previous reports confirm fibrotic capsules are minimal (1-3,5-8). Scant and focal chronic inflammatory cell reactions to ePTFE material may be explained by micro-motion at the tissue-implant interface, 

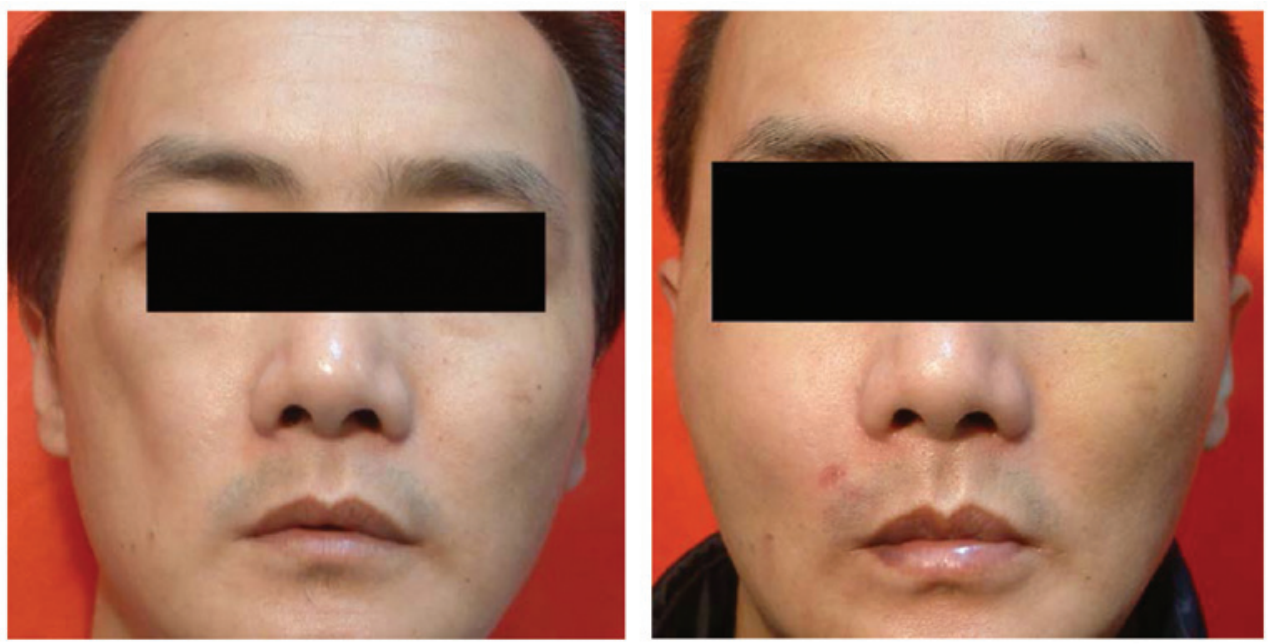

Figure 2. Expanded polytetrafluoroethylene implant augmentation, via preauricular incision, in a 36-year-old man with a major depressed deformity of the right cheek. Left, preoperatively; right, 7 months postoperatively.
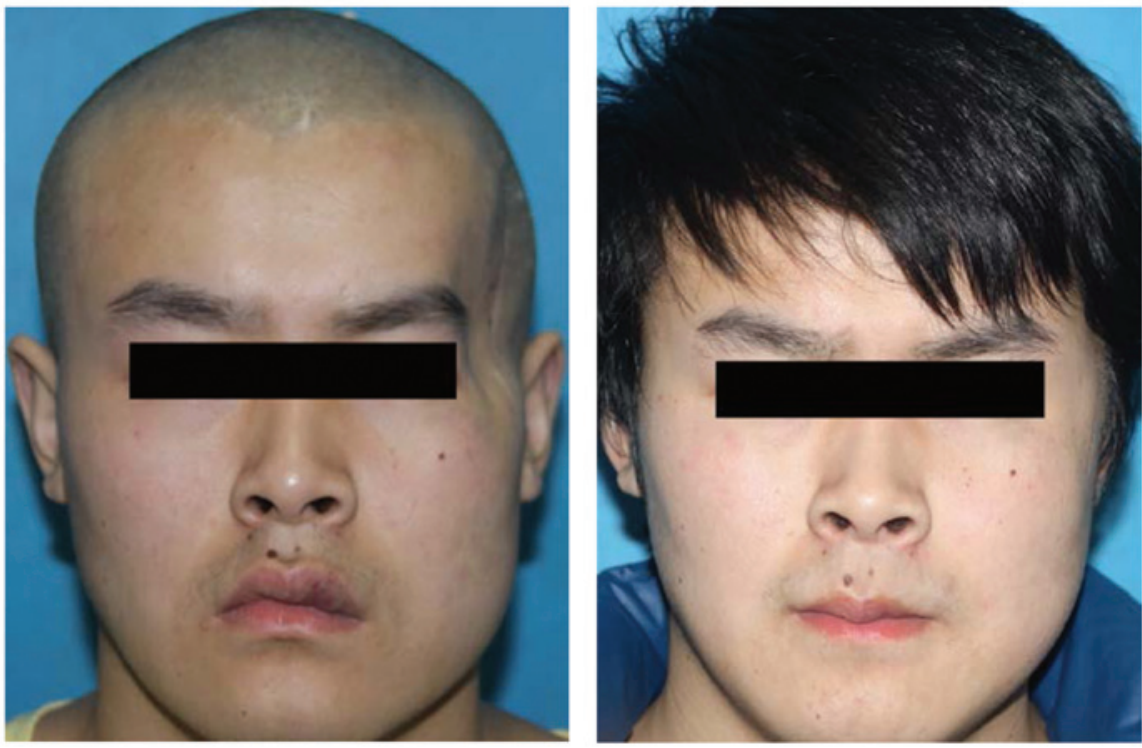

Figure 3. Expanded polytetrafluoroethylene material augmentation, via an incision behind the temporal hairline, in a 23 -year-old man with a depressed deformity of left temporal area. Left, preoperatively; right, 1 year postoperatively.
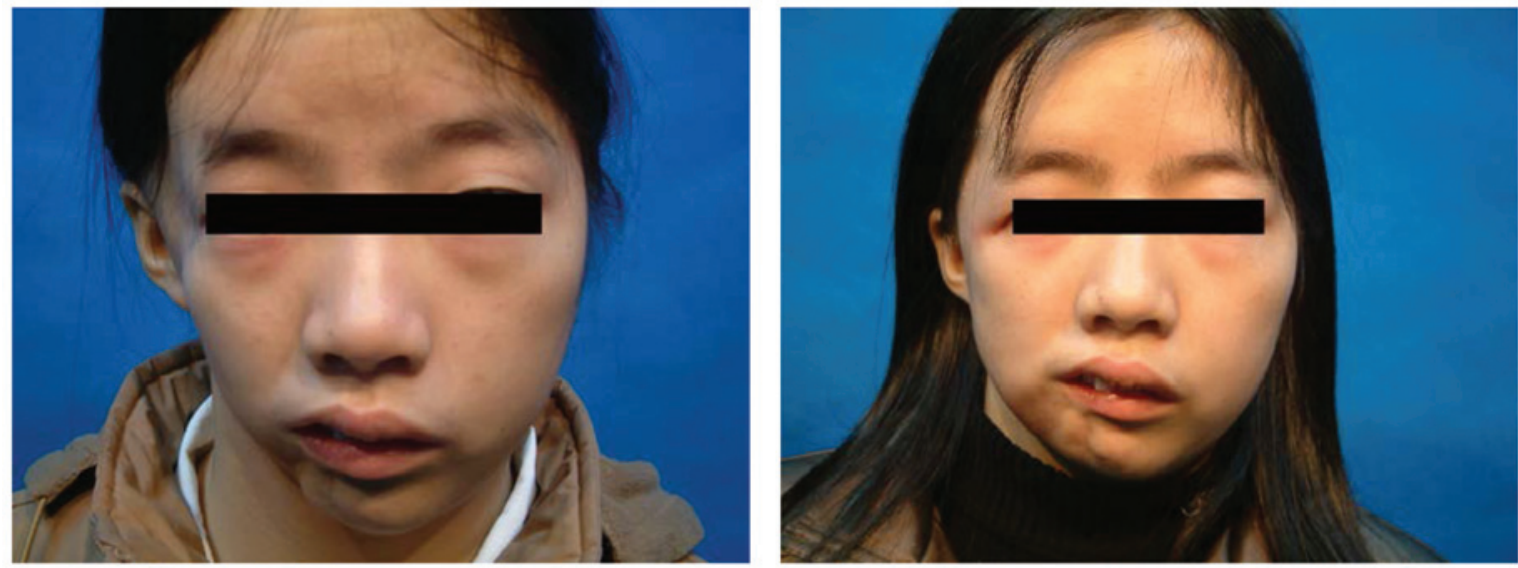

Figure 4. Multiple expanded polytetrafluoroethylene implants in a 25 -year-old woman with right facial depressed deformities from the pre-mandibular area to the temporal area. Implants were inserted into the tissue plane between the subcutaneous tissue and superficial musculoaponeurotic system in the pre-mandibular, cheek and zygomatic areas; and into the plane under the superficial temporal fascia, via an incision behind the temporal hairline. Left, preoperatively; right, 1 year and 3 months postoperatively. 
contamination, or reaction to the material configuration. However, the implant material itself does not appear to be a direct stimulus for inflammation, unlike Proplast, another form of polytetrafluoroethylene, which elicits an intense and ongoing inflammatory cell reaction that does not subside but rather markedly increases over time (4).

Various methods for correcting facial depressed deformities have been described in the literature $(6,9)$. Silicone and Gore-Tex are the most widely used implant materials for skeletal augmentation. However, certain intrinsic properties such as stiffness and molding difficulty, hinder their use in soft tissue augmentation. If the patient has a thin skin texture or the implant is placed in a dynamic expression rich area, the borders of the hard implant can be identified or palpated. Fat injections or autologous fat grafting for facial contouring are one of the most frequently employed methods as they are easy to perform and involve a relatively minor invasive procedure. However, autologous fat grafting has the critical pitfall of unpredictable resorption over time and the risk of uneven distribution of fat throughout the area that the surgeon wants to modify.

During the procedure, attention should be paid to the implanting tissue plane and implanting region. As mentioned earlier, aside from frontal and temporal regions, ePTFEs can be inserted into the tissue plane between the SMAS and subcutaneous tissue. Particularly important for such a procedure is to ensure the skin is sufficiently thick to conceal the traces of the edges of the ePTFE. It is not recommended to implant ePTFE materials in the regions of lips and nasolabial folds, however, as there is an increased risk of a discomforting firm or stiff feeling (10).

Biotolerability is defined as the ability of a material to reside in the body for long periods of time with only low degrees of inflammatory reaction (11). Various factors affect biotolerability, including the biomaterial itself and the implanting procedure. Bacterial infections and repeated frictions in the implantation regions are important contributing factors for inflammatory reactions. Thus, to increase biotolerability, we refined our operational procedures to decrease the possibility for inflammatory reactions. We stringently adhered to the principles of aseptic technique, avoided frequent placing in and removal from the dissected pocket, suturing the incision tightly away from potentially invading bacteria.

In conclusion, ePTFE is biocompatible and can be well tolerated by the host. Through mature preoperative planning and skillful manipulation, ePTFE can yield a good outcome. Aside from lipofilling and a free-flap transfer, ePTFE constitutes a viable alternative to consider for the treatment of depressed deformities of the face.

\section{Acknowledgements}

The present study was supported by the Chongqing Science and Technology Commission (Cstc2013kjrc-qnrc10003).

\section{References}

1. Cuzalina LA and Hlavacek MR: Complications of facial implants. Oral Maxillofac Surg Clin North Am 21: 91-104, vi-vii, 2009.

2. Sclafani AP and Romo T III: Biology and chemistry of facial implants. Facial Plast Surg 16: 3-6, 2000.

3. Rubin JP and Yaremchuk MJ: Complications and toxicities of implantable biomaterials used in facial reconstructive and aesthetic surgery: A comprehensive review of the literature. Plast Reconstr Surg 100: 1336-1353, 1997.

4. Maas CS, Merwin GE, Wilson J, Frey MD and Maves MD: Comparison of biomaterials for facial bone augmentation. Arch Otolaryngol Head Neck Surg 116: 551-556, 1990.

5. Maas CS, Gnepp DR and Bumpous J: Expanded polytetrafluoroethylene (Gore-Tex soft-tissue patch) in facial augmentation. Arch Otolaryngol Head Neck Surg 119: 1008-1014, 1993.

6. Artz JS and Dinner MI: The use of expanded polytetrafluoroethylene as a permanent filler and enhancer: An early report of experience. Ann Plast Surg 32: 457-462, 1994.

7. Schranz D, Jux C, Vogel M, Bauer J, Akintürk H and Valeske K: Large-diameter graft-stent (Advanta V12) implantation in various locations: Early results. Cardiol Young 21: 66-73, 2011.

8. Niamtu J III: Advanta ePTFE facial implants in cosmetic facial surgery. J Oral Maxillofac Surg 64: 543-549, 2006.

9. Baek R, Heo C and Kim BK: Use of various free flaps in progressive hemifacial atrophy. J Craniofac Surg 22: 2268-2271, 2011.

10. Cox SE: Who is still using expanded polytetrafluoroethylene? Dermatol Surg 31: 1613-1615, 2005.

11. Ratner BD: The biocompatibility manifesto: Biocompatibility for the twenty-first century. J Cardiovasc Transl Res 4: 523-527, 2011. 